\title{
Research Paper: Normalizing the Bender Visual-Motor Gestalt Test for 4 to 7 Years Old Children of Tehran, Iran
}

\author{
Siamak Tahmasebi ${ }^{1}$, *Saman Mafakheri Bashmaq ${ }^{2}$, Atefe Emad-al-din ${ }^{1}$, Rahele Rezaei ${ }^{1}$ \\ 1. Department of Preschool Education, University of Social Welfare and Rehabilitation Sciences, Tehran, Iran. \\ 2. Department of Psychology of Exceptional Children, Faculty of Psychology \& Education, Allameh Tabataba'i University, Tehran, Iran.
}

Citation: Tahmasebi S, Mafakheri Bashmaq S, Emad-al-din A, Rezaei R. [Normalizing the Bender Visual-Motor Gestalt Test for 4 to 7 years old children of Tehran, Iran (Persian)]. Journal of Rehabiltation. 2016; 17(1):20-31. http://dx.doi.org/10.20286/jrehab-170118

: http://dx.doi.org/10.20286/jrehab-170118

Received: 26 Apr. 2015 Accepted: 15 Sep. 2015
Keywords: Normalization, Bender Visual Motor Gestalt Test, Neuropsychological Tests, Perceptual motor performance, Preschool children

\section{ABSTRACT}

Objective Bender-Gestalt is one of the well-known neurocognitive tests designed by Lorta Bender to evaluate perceptual-motor development level in children. Besides its principle application in evaluation of cerebral damage, Bender test has other important applications, such as evaluating children for entering school, anticipating educational progress, diagnosing children with reading and learning disorders, studying growth retardation, as well as a nonverbal intelligence test to evaluate children's reading readiness to enroll in elementary school. Because of the importance of this test in many diagnostics aspects, it is necessary to prepare a normalized form of this test. In this study, we tried to provide this form in a more comprehensive and updated form compared to previous studies.

Materials \& Methods This is a descriptive-analytic research. The study population comprised all preschool children in Tehran. In this research, we surveyed 523 ( 271 boys and 252 girls) preschool children in 3 groups of 4-5 years old ( 200 children, 95 boys and 105 girls), 5-6 years old (199 children, 112 boys and 87 girls), and 6-7 years old ( 124 children, 64 boys and 60 girls) who were selected by cluster sampling method from some preschools centers in south, north, and center of Tehran during the spring of 2012. The inclusion criteria were having normal intelligence quotient and being in the range of preschool age. Obtained protocols were numbered on the basis of expanded Koppitz scoring system, and finally were analyzed using the Chi-squared test. Results Data analysis for 4-5 years old group revealed the result of $M(S D)$ as $14.75(1.82)$. The most frequent errors belonged to distortion in plans A, 7, and then 8. Also, the least frequent errors belonged to linear drawing of plans 3 and 6 . With regard to misproportion error in plan $A$ and repetition error in plan 2, girls made significantly less errors than boys, while with regard to spin errors of plan 7 and misproportion in plan 7, boys got the higher scores. In 5-6 years old group of children, M(SD) was 9.67(4.38). Also, the most frequent errors belonged to distortion in plans 8 and $A$. Furthermore, the least frequent errors belonged to linear drawing in plan 3, distortion in plan 7, and isproportion of components in plan 5. With regard to inappropriate composition and linear drawing of plan 5, boys made significant higher errors than girls. For children of 6-7 years old, $\mathrm{M}(\mathrm{SD})$ was 8.14(4.17). The most common errors in drawing belonged to first distortion errors in plans $A$ and 8 , and then spins in plans 7 and 3 , and lastly to misproportion in plan 7 . The least frequent errors belonged to linear drawing of plans 5 and 3, spin in plans 8 and 1, and finally distortion in plan 1 . Male gender was effective in committing errors of wrong composition of plans A and 3, spin in plan 1, figure distortion in plans 6 and 7 , also female gender in errors of wrong composition of plan 2, spin in plans 2 and 8.

Conclusion Consistent with Koppit'z maturational hypothesis and previous studies (Iranian and foreign), our results showed visual-motor improvement of perceptual performance with increasing age. In conclusion, koppitz scoring system is applicable to Iranian children protocols.

\footnotetext{
* Corresponding Author:
}

Saman Mafakheri Bashmaq, MSc.

Address: Department of Psychology of Exceptional Children, Faculty of Psychology \& Education, Allameh Tabataba'i University, Dehkadeh-ye-Olympic, Tehran, Iran.

Tel: +98 (918) 2268880

E-Mail: saman.mafakheri@yahoo.com 


\title{
هنجاريابى آزمون ديدارى-حركتى بِندر -تِشتالت در تروه كودكان f-V ساله تهران
}

\author{
سيامك طهماسبى'، "سامان مفاخرىباشماق'؛ عاطفه عمادالدين'، راحله رضايى' \\ ا - ك كروه رشد و يرورش كودكان ييشدبستانى، دانشعاه علوم بهزيستى و توانبخشى، تهران، ايران.

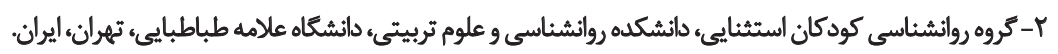

\begin{abstract}
حكبد

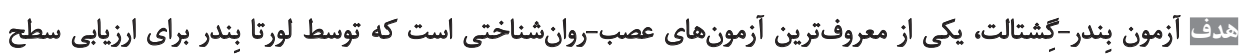

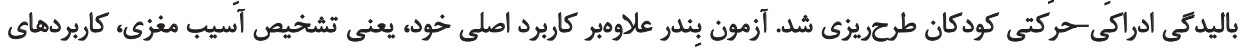

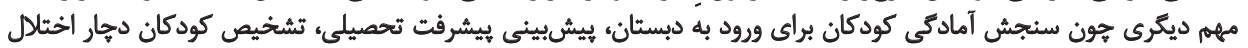

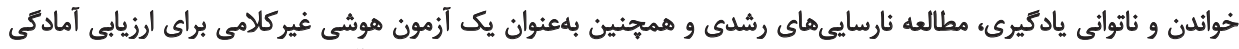

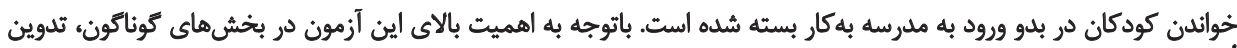

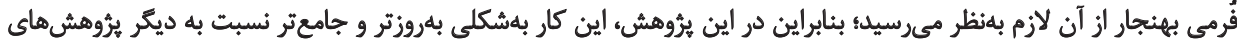

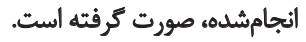

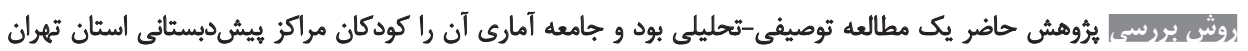

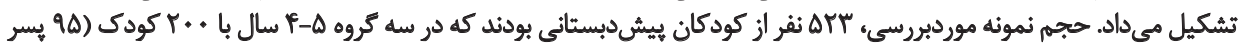

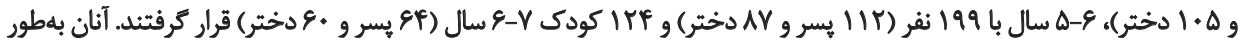

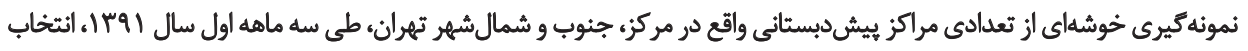

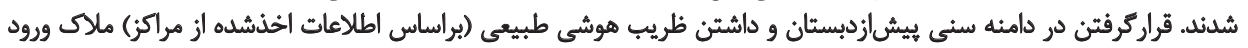

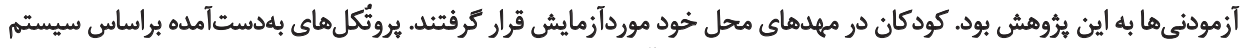

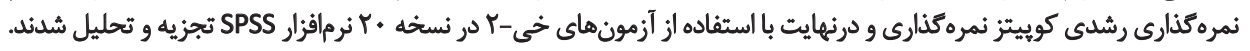

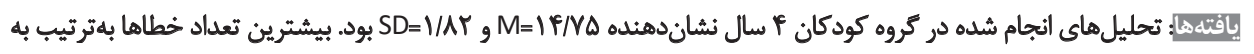

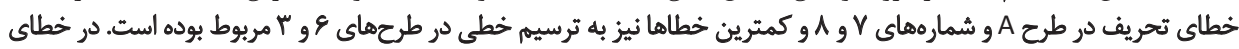

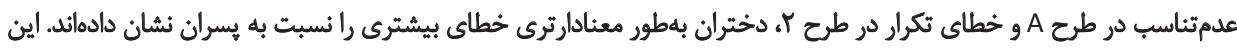

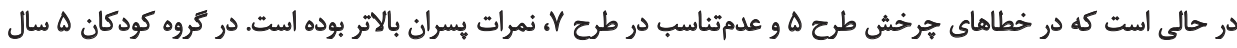

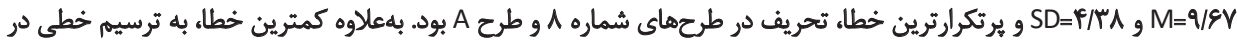

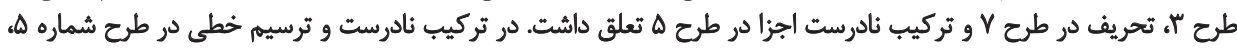

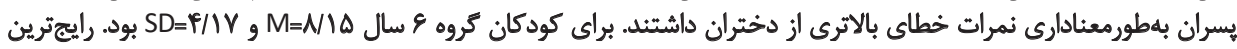

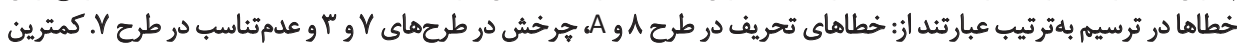

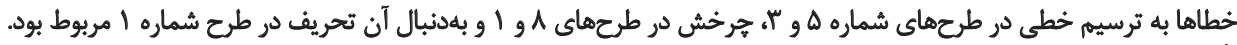

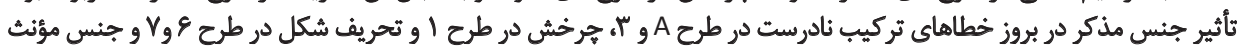

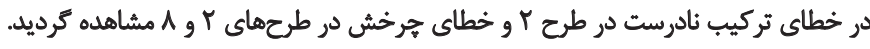

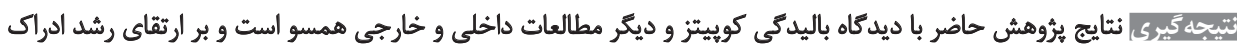

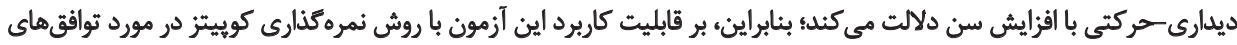
كودكان ايرانى اشاره دارد.
\end{abstract}

تاريخ دريافت: 4.ارديبيشت T9F

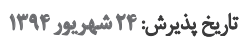

$$
\begin{aligned}
& \text { كليدواروها: } \\
& \text { هنجاريابى، آزمون } \\
& \text { ديداري - بركتي }
\end{aligned}
$$

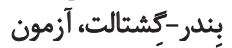

$$
\begin{aligned}
& \text { عصب-روانشناختى، } \\
& \text { عملكرد ادراكى حركتئي، } \\
& \text { كودكان ييشدبستاني }
\end{aligned}
$$


هماهنكّى ادراك ديدارى حركتي كاربرد دارد [9-ب].

مقلمه

بلهور كلى در كشور ما مهمترين مسائل فنى روانسنجي در كاربرد

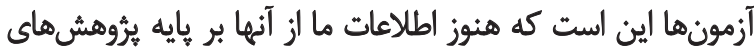

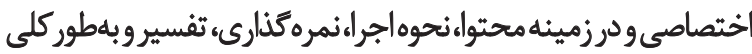

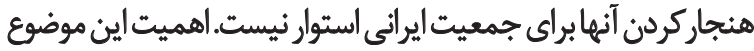

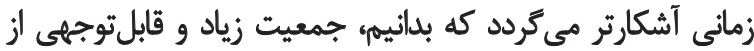

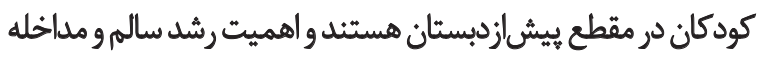

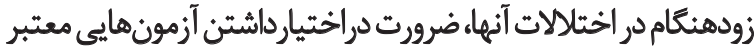

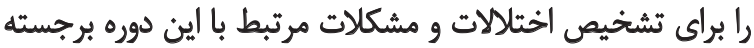

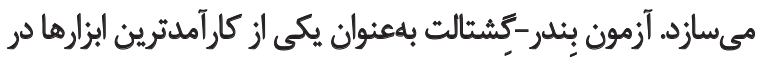

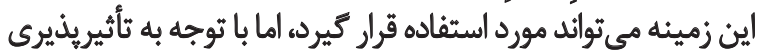

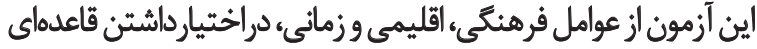

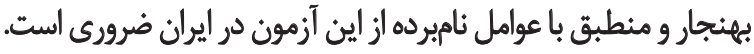

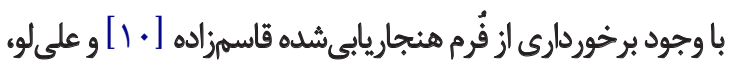

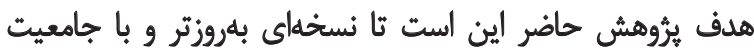

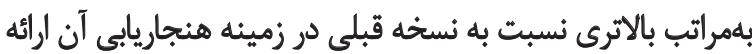

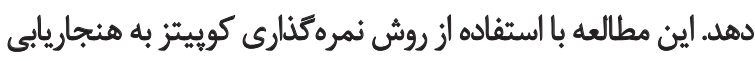

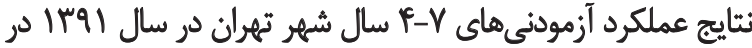

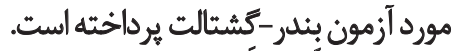

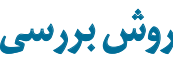

\section{جمعيث، ثمونه وروش ثمونليكيرى}

ريثروهش حاضر مطالعهاي توصيفى -تحليلى است و جامعه آمارى آن

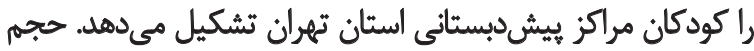

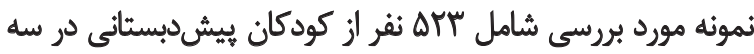

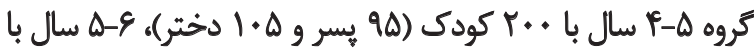

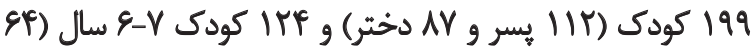

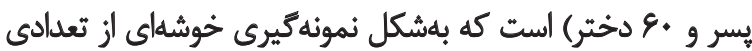

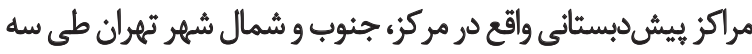
ماهه اول سال (9 إبا، انتخاب شدند.

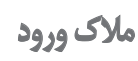

ملاك ورود آزمودنىها به اين يُؤوش را مواردى مائند قراركرفتن

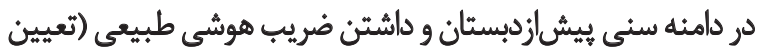

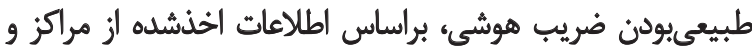

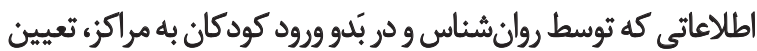

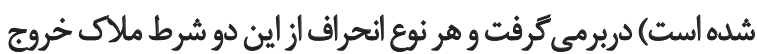

$$
\text { در نظر كرفته مى درئ. }
$$

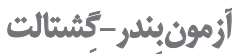

آزمون ديدارى حركتى "إبئدره بهعنوان ابزار غربالكرى براى براى

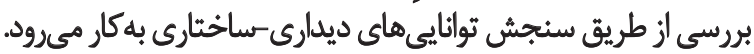

أزمون بندر-كَشتالت'، يكى از يركاربردترين آزمونهاى عصب-

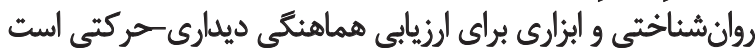

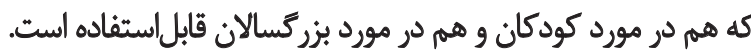

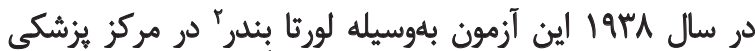

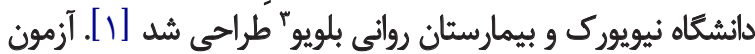

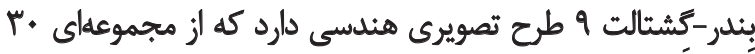

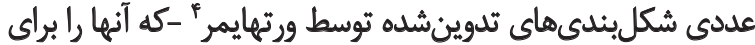

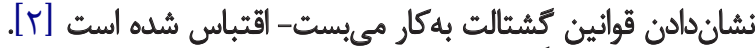

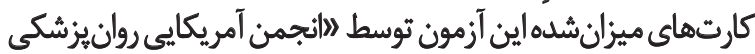

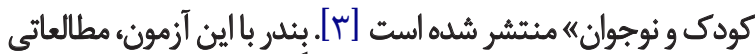

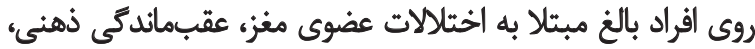
آفازي، يسيكوز، نوروز و تمارض انجام دادي

ورتهايمر بر تواثيي افراد بهنجار براى ياسخ به طرحها بهشيوهاي

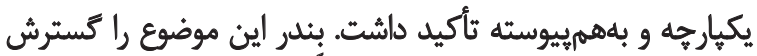

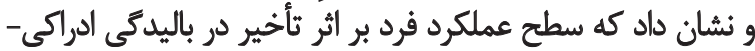

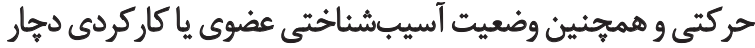

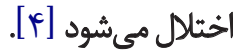

درحقيقت، بسيارى از بيماران مبتلا به آسيب مغزى از تحليل

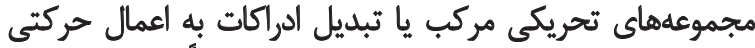

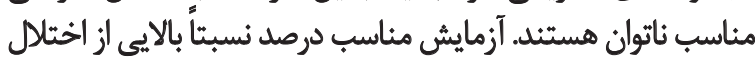

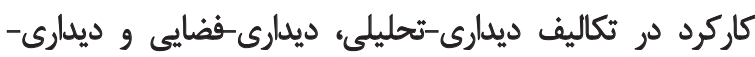

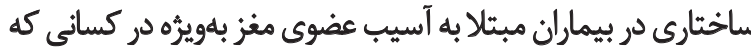

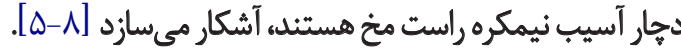

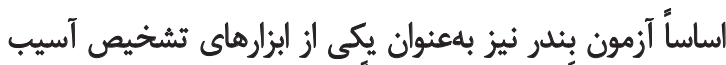

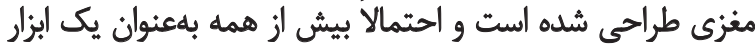

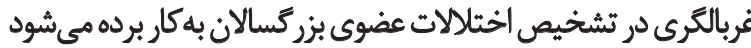

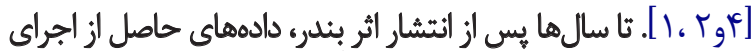

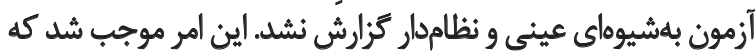
ارزشيابى آزمون دشوار شود [9]. از قديمىترين نظامهاى نمرهكذارى بزركسالان كه بهوكونهاي

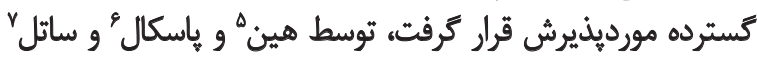

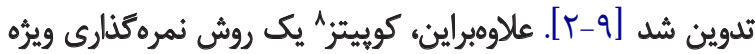

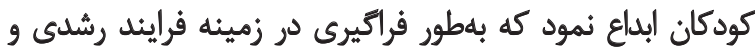

1. Bender-Gestalt test

2. Lorta Bender

3. Bellevue

4. Wertheimer

5. Hain

6. Pascal

7. Suttel

8. Koppitz 
اكرجه اين آزمون براى تشخيص آسيب مغزى بهكار ميرود، اما در

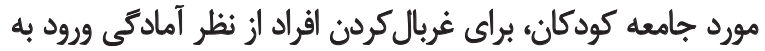

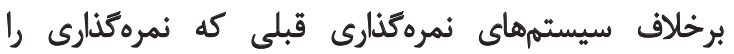

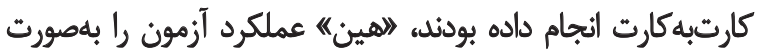

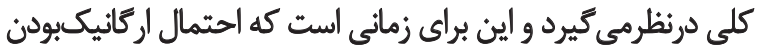

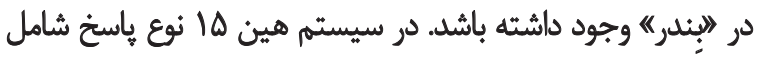

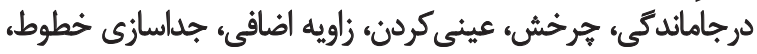

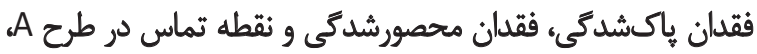

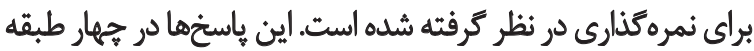

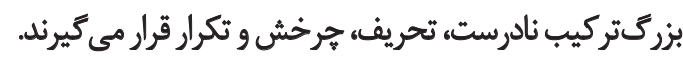

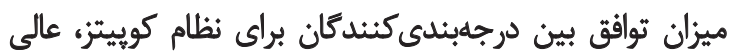

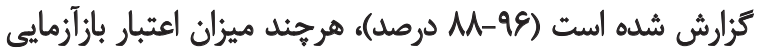

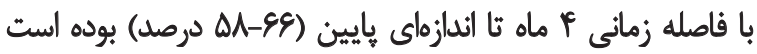

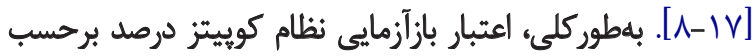

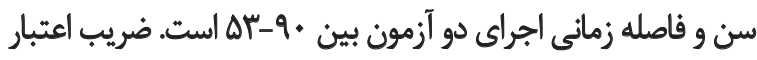

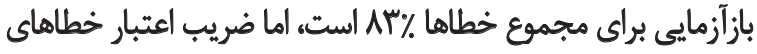
خاص بسياريايين وغيرقابل باعتماد بهحساب ميى آيد.

روش اجرا

بعد ازز ارائه آموزش هاى لازم درباره نحوه اجرا ونمره كذارى آزمون به بهازيه

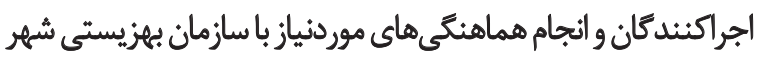

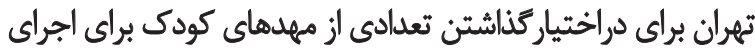

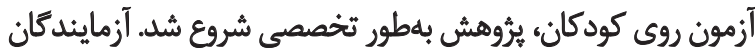

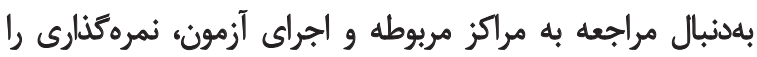

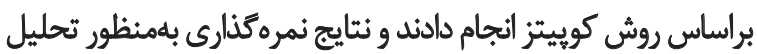
در اختيار همكاران طرح قرار كرفت.

تبجزيه و تحليل دادها

در يثرهش حاضر تجزيه و تحليل دادهها با استفاده از

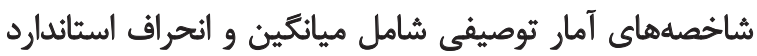

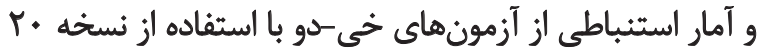

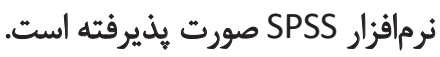

\section{اصول الخالاقى حاكم بر بثوهش}

اصول اخلاقى موردتوجه در اين يرؤهش عبارت بودند ازئ در حين

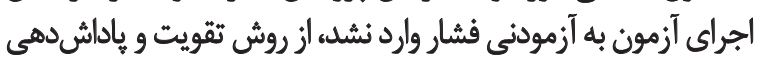

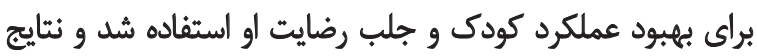

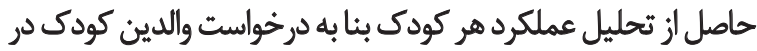

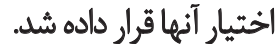

ياقتهما

يافتههاى يثروهش حاضر برمبناى اطلاعات بلهدست آمده از بخش

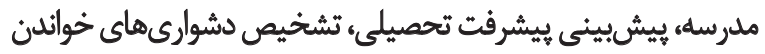

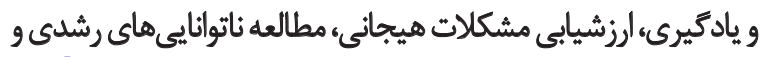

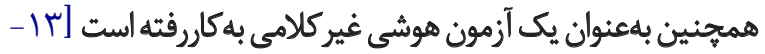

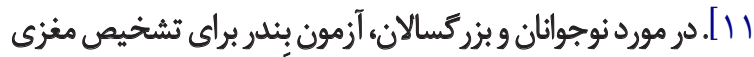

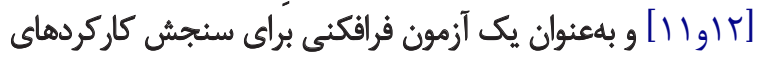

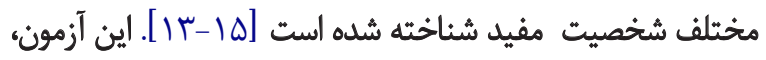

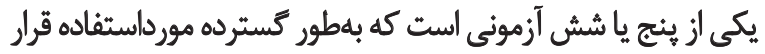

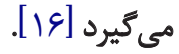

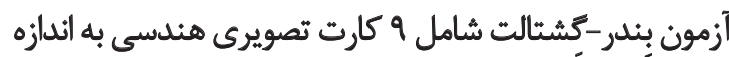

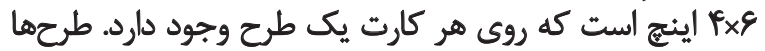

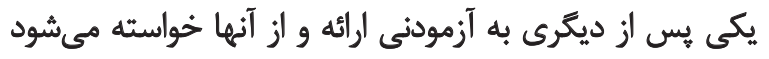

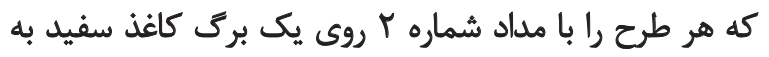

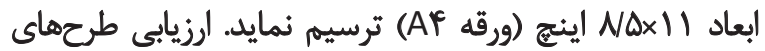

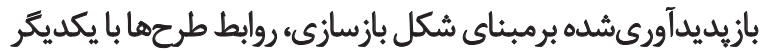

$$
\text { و زمينه فضايى كلى آنهاصورت مى كيرد بازي }
$$

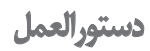

روش اجراى آزمون بهصورت فردى توسط هات، بهعنوان روش

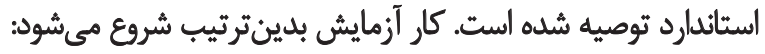

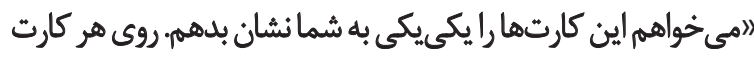

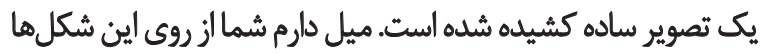

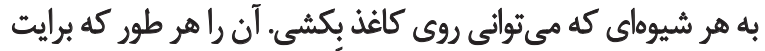

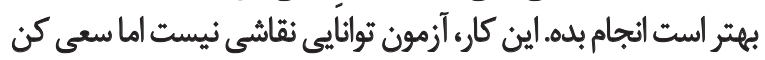

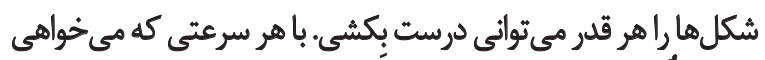

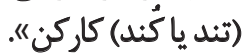
ابتدا كار A رادرحالي كه حرف A در قسمت بايين كارت قرار كرفتئه

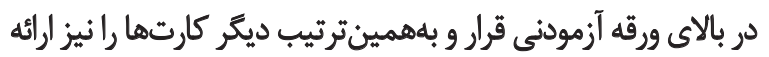

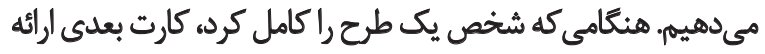

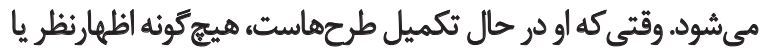

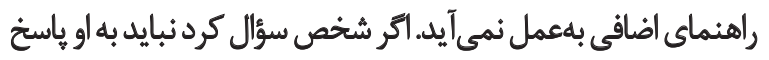

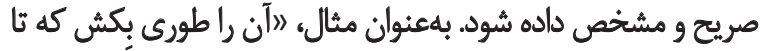
جاى ممكن شبيه عكس روى كارت باشده".

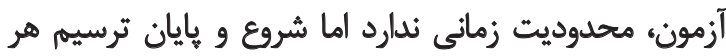

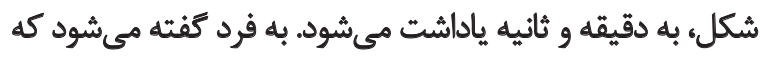

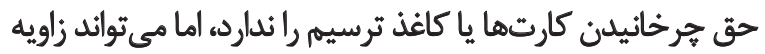

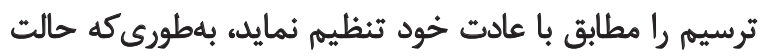

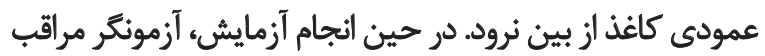

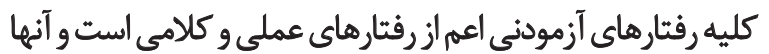

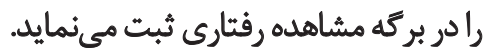
اجراى آزمون و تحليل آنها، در جداول ا، ب و بـ نشان داده شده است. 


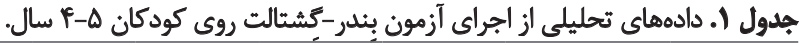

يسر

دختر

خى-Y ييرسون

تصحيح بيوستّى

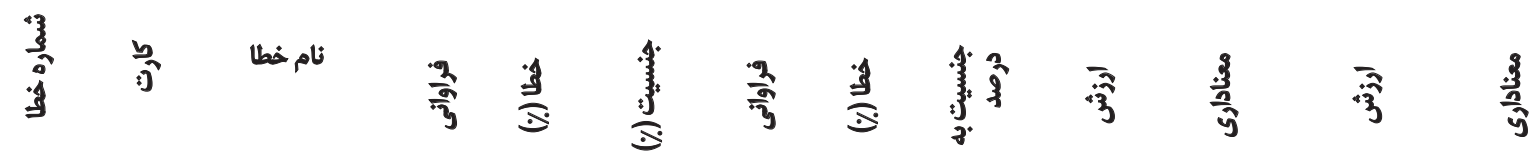

\begin{tabular}{|c|c|c|c|c|c|c|c|c|c|c|c|c|}
\hline 1 & \multirow{4}{*}{$A_{\text {r }}$} & تحريف شكل & 9. & $\mathrm{FV} / \mathrm{q}$ & $9 \Delta / V$ & Q & $\Delta T / 1$ & $9 / / \Delta$ &.$/ q \Delta Y$ & . & "TEM &.$/ F q$ \\
\hline r & & عدمتناسبب & rV & $r g / \Delta$ & TNY & PV & $\alpha \pi / \Delta$ & $\mu+/ \mu$ & $\Delta / T I T$ &.$/ 4 r$ & F/DFT & 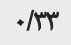 \\
\hline$r$ & & جرخش & $\Delta$. & $\Delta$ & $\Delta T / T$ & $\Delta$ & Q. & $\mathrm{PV} / \mathrm{r}$ & - & + $/ 49 \Delta$ & $.10 . r$ &.$/ 479$ \\
\hline$p$ & & تركيب نالدرست & D) & $P T / \Delta$ & $\Delta H / A$ & 89 & $\Delta V / \Delta$ & $\rho \Delta / 1$ & $T / N V$ &.$/ 1 P$. & $1 / V e A$ & . MAT" \\
\hline$\Delta$ & \multirow{3}{*}{ طرح } & تحريف شكل & ra & $r \Delta / T$ & $r+\mu$ & $p$ & $\Delta \varphi / A$ & $\mathrm{r} / \mathrm{A}$ &.$/ M \pi$ & . 6 Y9 &.$/ 118$ & $\cdot M T \Delta$ \\
\hline 8 & & $\stackrel{4}{\Rightarrow}$ & $M$ & $\varphi / /$ & $r \Delta / \Delta$ & $\pi$ & $\Delta V / q$ & rM & - NEY & $\cdot|r A|$ & . / $181 \mathrm{~V}$ & $.1 m$ \\
\hline$r$ & & ادامه كارى & vo & $\varphi g / \pi$ & $v / 1 /$ & AV & $\Delta r N$ & $A T / q$ & $\cdot / 2+9$ &.$/ \Delta V A$ & $.11 \% 9$ & $\cdot N \cdot 9$ \\
\hline 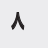 & \multirow{3}{*}{ ط } & شُه & $\pi$ & $\Delta \cdot / \Lambda$ & $r \Delta / 1$ & $\pi$ & $p q / r$ & $r \cdot \Delta$ & - rer & - MVAa &.$/ \pi q$ &.$/ \Delta N$ \\
\hline 9 & & تركيب نادرست & ge & $p q / r$ & $v \cdot \pi$ & \&s & $\Delta \cdot N$ & $8 * / 4$ & $.18 \mathrm{~V}$ & . Mir & - MPA & $\cdot 10 \cdot \Delta$ \\
\hline 1. & & ادامه كارى & $\pi$ & $r g / A$ & $m$ & $\Delta \Delta$ & $R / \pi$ & $\Delta T / T^{\circ}$ & EMV & .10 .9 & $8 / . \Delta \varphi^{\circ}$ & .1 .14 \\
\hline 11 & \multirow{4}{*}{ طرح } & تحريف شكل & ra & $P \mu / T$ & exp. & is & $\Delta \Delta / A$ & $P \Delta / \pi$ & 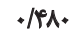 &.$/ 419$ & $\cdot r \cdot r$ & 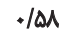 \\
\hline$\pi$ & & هرخ & $\pi$ & $p q / r$ & $r \Delta / 1$ & $\pi$ & $\Delta \cdot N$ & $\pi / 1$ & $\cdot \pi \cdot \Delta$ &. $\mid \& \Delta$ & .1 .94 & . NET \\
\hline $\mathbb{1 r}$ & & تركيب نادرست & ه) & $\mu / \mathrm{N}$ & $\Delta f / \pi$ & $g$ & $\Delta \Delta / \pi$ & $\Delta 9 / 4$ & 1. ब. &.$k 8$ &.$/ T \Delta t$ & - $/ \Delta Q Y$ \\
\hline if & & ترسيم خطى & $r_{0}$ & FNA & $r / \pi$ & $m$ & $\Delta 1 / \pi$ & $12 / 4$ & .1 .98 & - Mal & $\% v V$ & 1.948 \\
\hline 10 & \multirow{2}{*}{ طر } & مُش & $n$ & D. & $V \Delta / \Delta$ & $n$ & Q. & ov & INEA & . MAM & $1 / \pi / A$ &.$/ M E$ \\
\hline 19 & & تركيب نادرست & $m$ & $r \Delta / r$ & $r g / r$ & is & $\Delta F / N$ & mq &.$/ 1 Y a$ & 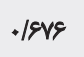 & $\% V^{e}$ & - NNE \\
\hline iv & \multirow{4}{*}{ طعه } & تحريف شكل & w & $r q / 1$ & YNV & rr & $8 . / 9$ & rq/s & $8 / 819$ & .11 .8 & $r / 14 Q$ &.$/ N F$ \\
\hline M & & هرخش & rv & $\Delta Q / V$ & $r q / e^{c}$ & ro & $r=/ r$ & $\mathrm{~m} / \mathrm{g}$ & Q/VaV & .1 .18 & $\Delta / \circ A \mu$ & A tor \\
\hline 19 & & تركيب ثادرست & M & $\Delta) / N$ & $\pi$ & pq & 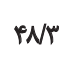 & $\mathrm{rV} / \mathrm{s}$ & - Meq & . RAY & .10 .9 & $.10 \mathrm{~W}$ \\
\hline r. & & ترسيم خطى & ra & $r \Delta / r$ & $41 / \pi$ & $\forall$ & $\Delta t / A$ & $\mu r / \mu^{\circ}$ & $\% / * M$ & - NeE & . & - /ArA \\
\hline r) & \multirow{4}{*}{ طح } & تحريف شكل & $\pi$ & $r \Delta / q$ & $W / 4$ & As & $\Delta * / /$ & $A \cdot / r$ & . MTA & $.18 \pi$ & .1 .94 & . NeI \\
\hline$\pi$ & & تركيب ثادرست & $\pi$ & $\Delta 1 / 8$ & $\pi$ & r. & $P N{ }^{*}$ & $r N T$ & . NFA & $\cdot|T \wedge|$ & . &.$/ p r$ \\
\hline M & & ترسيم خطى & rf & $\Delta T / T$ & $r \Delta / \Delta$ & $m$ & FON & $r$. & INAY & - MAT &.$/ \Delta A$ & ./pee \\
\hline$\eta$ & & ادامه كارى & $\mu$ & $F \in / V$ & $r \Delta / V$ & $p q$ & $\Delta t / N$ & $P \in N$ &.$/ .1 \mathrm{~V}$ &.$/ 198$ & $\%$ & $1 / \ldots$ \\
\hline ro & \multirow{4}{*}{$v$} & تحريف شكل & A & $x \in \|^{\circ}$ & $M^{2}$ & $q$ & $\Delta H / s$ & 9.18 & $\cdot / \pi{ }^{2}$ & $\cdot 19+Y$ & $\% 1 \%$ & $\cdot|m|$ \\
\hline 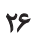 & & عدمتئاسب & me & 9. & $r \Delta / \Delta$ & 19 & $r=$ & $10 / 1$ & ए/\%q & .1 .98 & $T / M I$ & .1 .98 \\
\hline $\mathrm{TV}$ & & هرخش & or & FNT & $V / / r$ & $n$ & $\Delta V / \mathbb{A}$ & $8 V / q$ & . Tex & $\cdot 19 \cdot 7$ & $+/ N$ & $\cdot / M 19$ \\
\hline MA & & تركيب نادرست & 9 & $\mu e / \mu^{e}$ & ov & ra & $\Delta \Delta / \&$ & $n \in / \Delta$ & $1 /$ Tex & . & $1 / \pi$ &.$M M$ \\
\hline rq & \multirow{2}{*}{ طح } & تحريف شكل & 19 & $P N \mid$ & $9 \% / V$ & 98 & $\Delta 1 / 9$ & 9.18 & $1 / 418$ & $\cdot / \pi V$ & .1990 & $\cdot / f+t^{e}$ \\
\hline r. & & هرخش & M & $\Delta T / \Delta$ & $T / \pi$ & 19 & $\mathrm{PV} / \mathrm{\Delta}$ & $1 \mathrm{~V} / \mathrm{q}$ & $+18+Y$ & | & זים"א. & . IAPV \\
\hline
\end{tabular}


جدول r. دادههاي تحليلى از اجراي آزمون بندر -كشيتالت روى كودكان ؟-ه سال. دختر

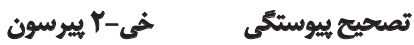

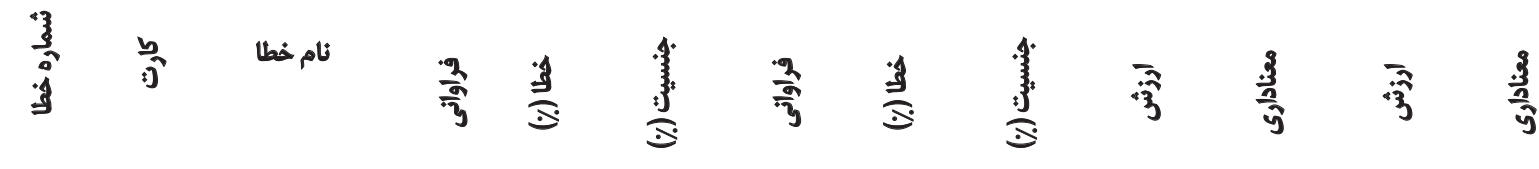

\begin{tabular}{|c|c|c|c|c|c|c|c|c|c|c|c|c|}
\hline 1 & & تحريف شكل & 81 & $\Delta \Delta$ & $r / \Delta r$ & Q. & Po & $\Delta / \Delta V$ & ./1V9 & $.8 n$ &.$/ . V A$ & . $/ \mathrm{AA}$ \\
\hline$r$ & & عدمثناسب & $\mathrm{rV}$ & of &.$/ T$ & r & eq & $8 / T^{\circ}$ & . NPY & $\cdot N \cdot r$ &.$/ \cdot \Delta f^{5}$ & $\cdot|A| S$ \\
\hline r & A b & هرخش & iv & $\Delta V$ &.$/ F T$ & ro & it & $r / f$. & .1 .81 & $\cdot / \Lambda \cdot \Delta$ & .1 .1 &.$/ 919$ \\
\hline$f$ & & تركيب نادرست & PI & $\Delta \Delta$ & $8 /{ }^{2}$ & $m$ & fo & $1 / 49$ & . IrA & 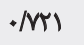 & .1 .94 & - Am \\
\hline$\Delta$ & & تحريف شكل & in & x & s/re & Tr & $r v$ & F/TE & $r / M T$ &.$/ r A$ & 1/An &.$/ 1 \%$ \\
\hline 8 & طع & هُهُش & $r$ & so & $\Delta / r$ & ir & ra & NIT & V/are &.$/ M I Q$ & $1 / 1.8$ & . \\
\hline Y & & ادامه كارى & re & $\Delta \Delta$ & $1 / \pi T$ & pq & 10 & $r / \pi$ & $\% \pi$ & - / 109 & $.1 . .1$ &.$/ 4$. \\
\hline$\wedge$ & & هرخش & Pr & er & $r / \% A$ & $\varphi$ & ץA & $9 / 7 q$ & $V / A F \Delta$ &.$/ M I I$ & I/TIY & $. / T Y \mid$ \\
\hline 9 & طرح & تركيب نادرست & Yo & 18 & $r / p V$ & Hi & $\Delta f$ & lar & $.1 . .1$ &.$/ 9 V A$ & 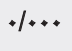 & $1 / \ldots$ \\
\hline 1. & & ادامه كارى & r. & Q. & V/IV & r. & $\Delta$. & . & $\cdot / A \cdot T$ &.$/ T V$ &.$/ 010$ &.$/ F r$ \\
\hline 11 & & تحريف شكل & re & 80 & $T / \pi T$ & if & TQ & $1 / 18$ & VAPV &.$/ M I F$ & $1 / 1+4$ & - /rra \\
\hline ir & $\mu=1$ & هرخش & TA & ar & $r / T r$ & $\pi$ & in & $F / T E$ & - $/ 4 \Delta r$ &.$/ \Delta .1$ &.$/ T Q F$ & .814 \\
\hline זו & 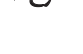 & تركيب نادرست & $\Delta f^{f}$ & $\Delta Y$ & $r / \& A$ & PI & rit & l/er & $.1 \cdot \pi$ & . 1 Av9 & $\% \ldots$ & ./994 \\
\hline if & & ترسيه خطى & $r$ & 1 & $V / r$ & . & - & $\%$ & Y/RES & / IFf & .19 .8 & 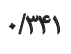 \\
\hline 10 & ط & هرخش & Qq & $\Delta V$ & V/Or & 10 & ז & $V(\Delta)$ & $.1 \cdot 11$ & -/1195 & $\%$ & $1 / \ldots$ \\
\hline if & 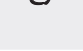 & تركيب نادرست & $\mu$ & $\Delta V$ & F/゙ & $\pi$ & Tr & NYE & $\% . \Delta f$ & $\cdot|\lambda| \varepsilon$ & $* 1+* V$ &.$/ 9 T Y$ \\
\hline IV & & تحريف شكل & YA & $\Delta r$ & . $12 \Delta$ & To & iv & V/TA &.$/ N A$ & ./QAF &.$/ 110$ & .1898 \\
\hline IA & & هرخش & il & pr & $N M$ & זr & $\Delta r$ & P/TE & V/FA. &.$/ 190$ & $1 /$ TE &.$/ \% 91$ \\
\hline 19 & 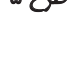 & تركيب نادرست & H & $n$ & $9 / 19$ & 1 & Tr & $r / 9$ & $r / / n^{e}$ & $+1+41$ & $r / \% 4$ & .1 .90 \\
\hline r. & & ترسيه خطى & rir & $n$ & $\Delta / r+$ & 9 & YA & $m /$ & r/NEA & $* / * \Delta Y$ & $r /+\Delta)$ & $*|+1|$ \\
\hline Y & & تحريف شكل & $r$ & $\Delta r$ & $\Delta / r+$ & r. & iv &.$/ \pi$ &.$/ 1 V^{\prime}$ & $.18 W$ & $.1 . \Delta 9$ & $\cdot 11+1$ \\
\hline r & & تركيب نادرست & 19 & Q1 & $+/ \mathrm{V}$ & 11 & $p q$ & $V / r$. &.$/ 4 r q$ & $+1 \Delta+r$ &.$/ \pi r$ & $.19 T \mathrm{~V}$ \\
\hline$\pi$ & $\infty$ & ترسيم خطى & r. & 9 & NYE & 11 & $r$ & $V / r$. &.$/ 997$ & $+/ 419$ & .1819 & $+/ 4+9$ \\
\hline MF & & الدامه كارى & PI & q. & $9 / 48$ & TV & fo &.$/ \mu$ & . I8V8 &. $\mid+11$ &.$|F a|$ & $\cdot / \Delta \cdot Y$ \\
\hline rA & & تحريف شكل & 19 & \&A & $+/ N Y$ & 9 & $\pi$ & $r /$ & $1 / N \Delta$ & •/MN & $1 / 499$ & $+\pi \varepsilon_{0}$ \\
\hline re & & عدمتناسب & 98 & $\Delta Y$ & $\sqrt[V]{ } \Delta \mathbb{A}$ & $p q$ & $\pi$ & $r / \Delta E$ & צ" / & + MIr & $+1+\Delta$ & . ATr \\
\hline TV & bح & هرخ & 89 & $\Delta \Delta$ & $1 / 41$ & 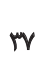 & to & $\Delta / F T$ & $.1 \cdot \pi$ & •/AHE & $+1++4$ & $+/ 941$ \\
\hline rA & & تركيب ثادرست & 81 & $\Delta q$ & $\Delta / \Delta F$ & ז" & il & $r / F q$ & . / 199 & $+/ * \lambda+$ & . MIV & $+/ \Delta n^{5}$ \\
\hline rq & $A_{z}$ & تحريف شكل & A. & $\Delta q$ & $F / M$ & $\Delta F$ & il & $F / 94$ & V/IrA & $+/ T M$ & - ATE & . \\
\hline r. & & هرخش هر & $r$. & $\Delta Q$ & V/IV & 18 & tr & $r / M$ & $.1 . .9$ & מוד & $+1+$. & $1 / \ldots$ \\
\hline
\end{tabular}


جدول "ا. دادهاى تحليلى از اجراي آزمون بندر-كشتالت روى كودكان Y-8 سال.

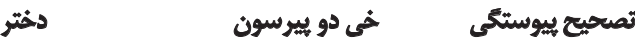

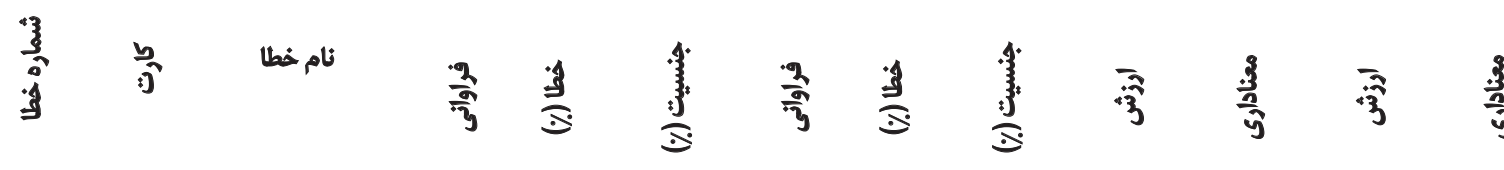

\begin{tabular}{|c|c|c|c|c|c|c|c|c|c|c|c|c|}
\hline 1 & \multirow{4}{*}{ ط ط } & تحريف شكل & A) & $\Delta A$ & rq/.8 & $\Delta$ & Pr & TNTT & r/TE. & 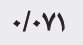 & r/RaP & $\cdot 11.1$ \\
\hline$r$ & & علدمثناسب & ir & ar & $1 . / 94$ & Mr & PA & 1. & $\cdot / N \Delta$ & - var & .1 .11 &.$/ 911$ \\
\hline$r$ & & مرخ & $\Delta q$ & $\Delta$ & $P \Delta / N$ & Pr & Pr & pelps & $1 / M$ & - Mar & ITVE &.$M P 1$ \\
\hline r & & تركيب نادرست & ir & ex & $\mid r / \Delta$. & rT & $r e$ & W/ES & $\Delta / r+r$ & $0 . r \mid$ & PIFIY & $.1 . M$ \\
\hline$\Delta$ & \multirow{3}{*}{ طرع } & تحريف شكل & 19 & of & PIEA & ro & $p$ & 1. & 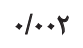 & .1989 & 100 & $1 / \ldots$ \\
\hline 8 & & جر & 18 & $n$ & $9 / r V$ & 8 & $r r$ & T/T & $r / \Delta N$ & 1.8. & $r \cdot V e$ & .1 .94 \\
\hline$v$ & & ادامه كارى & ro & $\Delta$ & ro & ra & if & ra &.$/ 799$ & - IOAF &.$/ 8$. & . $/ 819$ \\
\hline 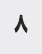 & \multirow{3}{*}{ طرح } & خه & $r e$ & $\Delta$ & PQ/FA & re & rr & rils & . & . ATE &.$/ 4 i$ & - /QTr \\
\hline 9 & & تركيب نادرست & rq & ro & ro & $n$ & ED & 10 & VNN & $\% \Delta$ & $V / . . V$ & .10 .1 \\
\hline 1. & & ادامه كارى & rq & ru & $10 / \%$ & $n$ & $n$ & $\pi / T$ & .1 .11 & -/A9T & .10 & $1 / \ldots$ \\
\hline 11 & \multirow{4}{*}{ ط } & تحريف شكل & $r 9$ & Po & $I V / M$ & pe & $\Delta \Delta$ & $r$. & r/4१q & $.1 * 4$ & refry & $.1 .9 x$ \\
\hline ir & & حرثش & 80 & $\Delta V$ & $\pi / A 1$ & 19 & $M$ & D) & $V / A Y Y$ &.$/ r q 4$ & $\cdot \mid A \cdot 1$ & $\cdot / r r$ \\
\hline ir & & تركيب نادرست & eq & e. & $r \cdot / r i$ & Po & f. & WTI & F/aAV & $\%$.re & F/M. & $\%$. r \\
\hline 1f & & ترسيم خطى & $r$ & EV & F/EA & 1 & $n$ & $r / \pi$ &.$/ 199$ &.$/ F \Delta \Delta$ & .100 & $1 / \ldots$ \\
\hline 10 & \multirow[b]{2}{*}{ ba } & مرثش & ar & $\Delta V$ & ro & Pr & $\pi$ & files & . $\mathrm{rar}$ & $\cdot \pi r$ & .1009 &.$/ F \Delta a$ \\
\hline 18 & & تركيب نادرست & ar & $\Delta 9$ & YO & 19 & m & ro & $r / T \cdot 4$ & ./Nrq & I/M & $* / / 8$ \\
\hline iv & \multirow{4}{*}{ bح } & تحريف شكل & r. & Pl & ro & $P r$ & $\Delta r$ & rlase & I/QYT &.$/ N A T$ & VOASE &.$/ r i r$ \\
\hline M & & جرخ & $M$ & $\mu$ & $9 / \pi$ & il & $\Delta$ & MVES & F/AFT & $\%$ H & $P / T \cdot A$ & $\% *$ \\
\hline 19 & & تركيب نالدرست & M & 81 & $9 / \pi r$ & $r+$ & rq & $1 / 89$ & I/riv &.$/ T 01$ & .1987 & . MTA \\
\hline r. & & ترسيهم خطى & $r$ & Q. & E/TQ & $r$ & a. & - &.$/ .40$ & $\cdot / A N^{2}$ & .10. & $1 / \ldots$ \\
\hline r & \multirow{4}{*}{ طرح } & تحريف شكل & af & 81 & $\Delta F / g A$ & il & rq & 198198 & F/RT & .1 .41 & P/.TE & $.1 .+\infty$ \\
\hline r & & تركيب نادرست & ri & $\varepsilon_{\Delta}$ & $10 / \%$ & ir & ra & 9189 & $r / r)$. &.$/ I T r$ & Iray &.$/ 194$ \\
\hline r & & ترسيم خطى & pe & $\Delta V$ & $r / M r$ & $m$ & $\pi$ & "זM & $\cdot N \cdot V$ &.$/ 4 *$. & - hat &.$/ F M$ \\
\hline rf & & ادامه كارى & 18 & $\Delta V$ & $M / N \Delta$ & Ir & gr & 10 & ./Ir & - MIr & $\% \mathrm{HY}$ & HNO9 \\
\hline Yo & \multirow{4}{*}{$v_{\tau}$} & تحريف شكل & $\pi$ & ov & $A F / T r$ & 19 & $r$ & $n \pi / T$ & $P / M N$ & $\%$ \% & $p / v q$ &.$/ . H T$ \\
\hline re & & علمتثناسب & 97 & $\Delta \Delta$ & $\mid r / \Delta$. & VD & ro & 1. & $M T$ &.$/ 290$ &.$/ 199$ & . $/ \Delta T A$ \\
\hline rv & & جرخش & r. & $\Delta \Delta$ & VD & $\Delta V$ & ro & $1 \Delta$ & . MIY & $.197 T$ & .1 .94 & $+N \Delta \Delta$ \\
\hline YA & & تركيب نادرست & or & $\Delta / \Delta$ & ENVD & $\Delta F$ & $\Delta / F q$ & $\gamma_{*}$ & I/TOS & . MFa & $1 /+4 \cdot$ & $\left.+/ x^{2}\right)$ \\
\hline rq & \multirow{2}{*}{ طح } & تحريف شكل & $q$ & $\Delta \Delta$ & VNIT & vq & Po & $9 \pi / T T$ & $+M \lambda$ &.$/ 1994$ &.$/ M \Delta$ & $+/ \Delta \Delta F$ \\
\hline r. & & هرخش & 11 & ג & S/TA & $M$ & ar & NMT & $r / \Delta T$ & .1 .81 & $r / A \cdot r$ & +1.94 \\
\hline
\end{tabular}




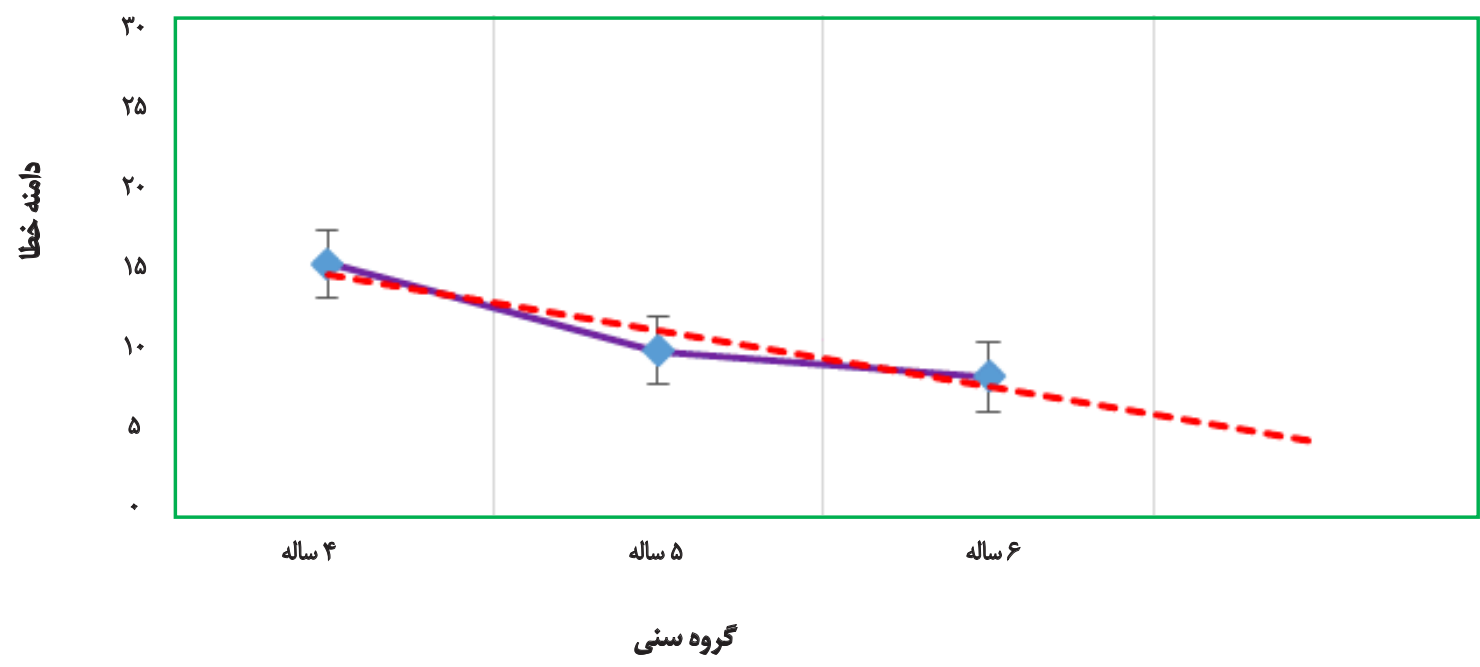

توانبخننى

تصوير ا. ميانكين نمرات آزمون بِندر -كشتالت در بررسى اخير.

براساس جدول شماره ץ، در كروه كودكان \&-ه سال نيز بيشترين

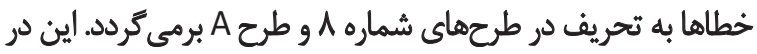

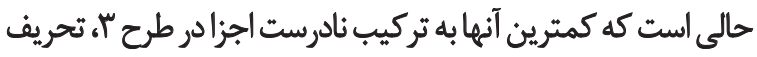

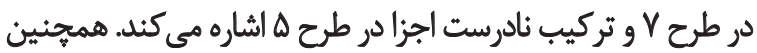

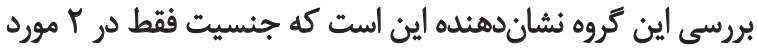

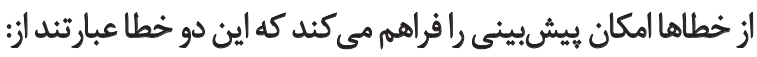
تركيب نادرست و ترسيم خطلى در طرح شماره ها كه در هر دو مورد

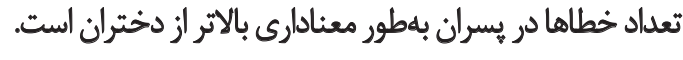
براساس جدول شماره به در كروه كودكان V-9 سال نيز رايجترين

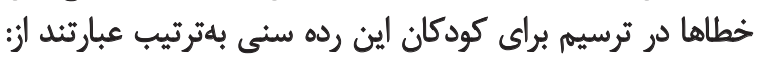

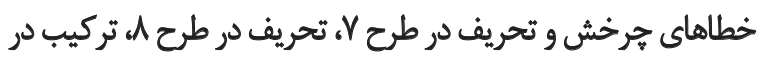

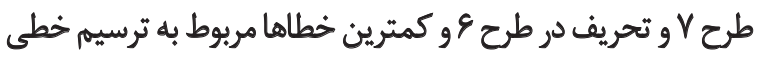

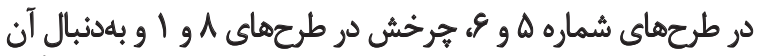

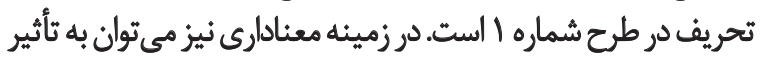

همان كونه كه در تصوير شماره ا نشان داده شده استه بين نمرات

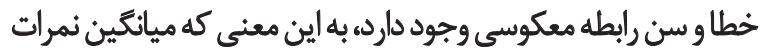

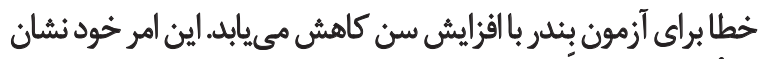

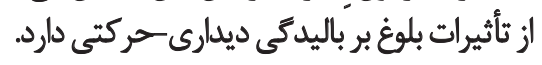

\section{ث:}

براساس جدول شماره ا، در كودكان ه-

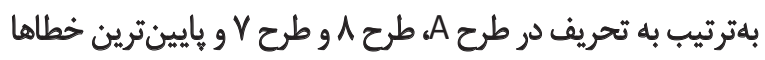

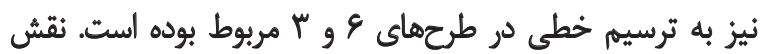
تفاوتهاى جنسيتى در خطاى ترسيم در اين كروه نيز به به اين شكل

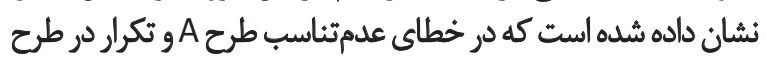

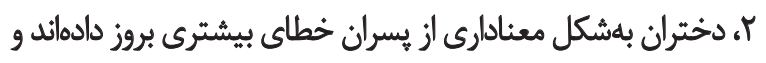

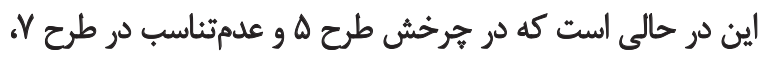

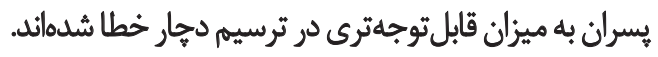

جدول f. مقايسه نثّايج تحقيقات هنجاريابى آزمون بِّدر.

\begin{tabular}{|c|c|c|c|c|c|c|c|c|c|c|}
\hline \multicolumn{3}{|c|}{ ئؤوشش حاضر (r+11) } & \multicolumn{3}{|c|}{ "*نجاريابى تهران (1 MMM) } & \multicolumn{3}{|c|}{ (I AVP) هنجاريابى آمريكا" } & \multirow[b]{2}{*}{ تيروه سنى } & \\
\hline SD & M & $\mathbf{N}$ & SD & M & $\mathbf{N}$ & SD & M & $\mathbf{N}$ & & \\
\hline l/Ar & IF/NA & $r+$. & - & - & - & - & - & - & $\begin{array}{l}f-p / 8 \mathrm{~m} \\
r / 8-\Delta \mathrm{m}\end{array}$ & r \\
\hline$r / H$ & QRV & 199 & 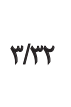 & $1 \pi / 9 \pi$ & if & $\begin{array}{l}r / r \\
r / \mu\end{array}$ & $\begin{array}{l}\mid W / 1 \\
9 N\end{array}$ & $\begin{array}{l}\text { PV } \\
\text { Ir. }\end{array}$ & $\begin{array}{l}\Delta-\Delta / 8 \mathrm{~m} \\
\Delta / \xi_{-} / \mathrm{m}\end{array}$ & $\Delta$ \\
\hline$P /$ Pr & NID & in & T/M & V/re & 19 & $\begin{array}{l}r / r \\
r / \Delta\end{array}$ & $\begin{array}{l}\text { NE } \\
V / r\end{array}$ & $\begin{array}{l}\text { IVD } \\
9 .\end{array}$ & $\begin{array}{l}8-9 / 8 \mathrm{~m} \\
\text { s/8-V m }\end{array}$ & \& \\
\hline
\end{tabular}

توانبخننى

"نقل از كراث-مارئات (19AF) [ه]

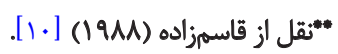




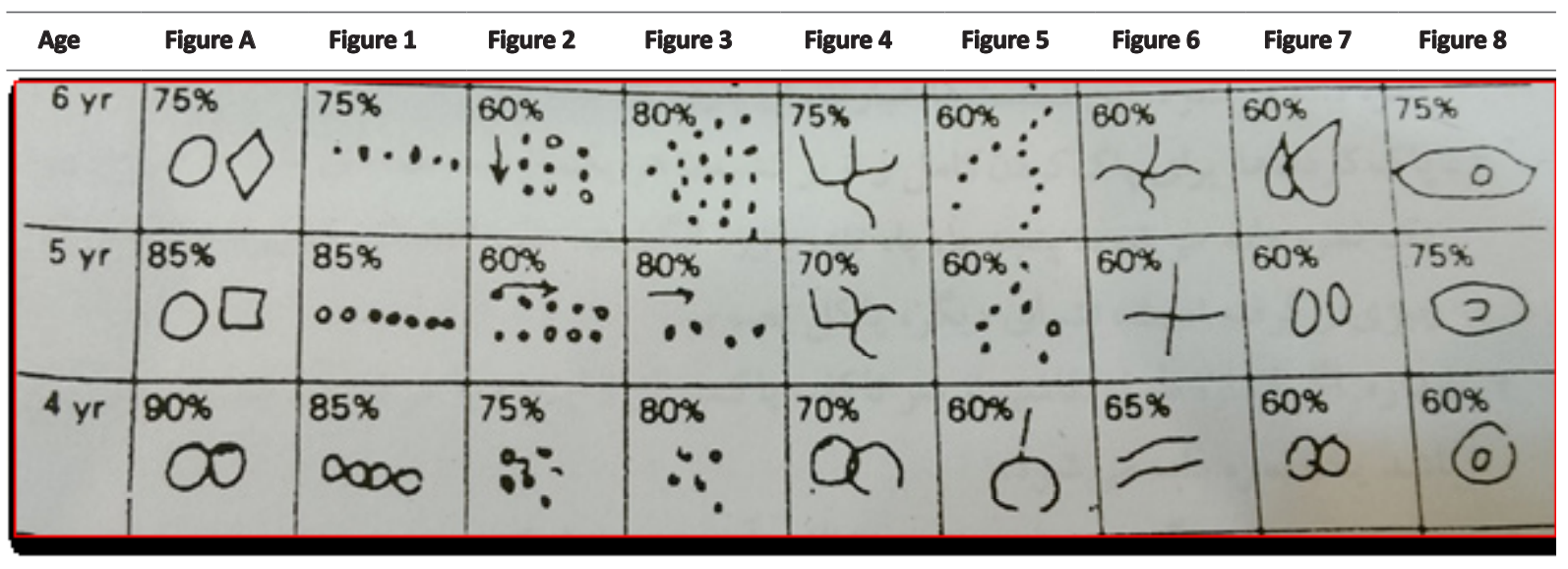

توانبخننى

تصوير Y. راهنماهاي رشلى براى طرحهاي بئلر-كُشتالت.

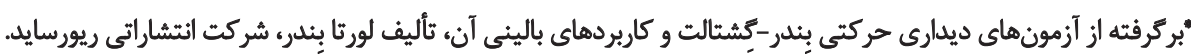

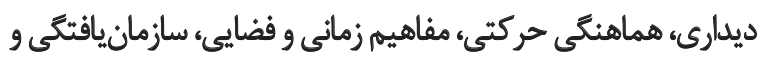

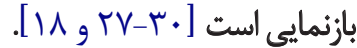

بلنظر مىرسد كه وحدت ديدارى حركتى بستكى به رشد و توسعه

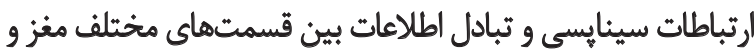

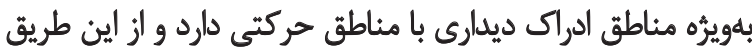

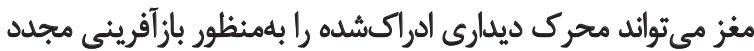

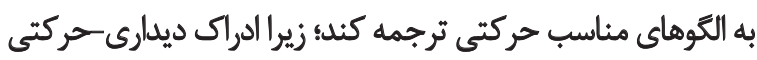

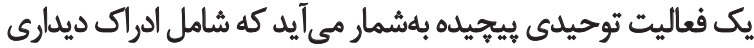

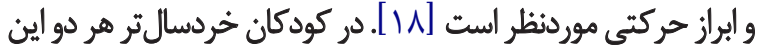

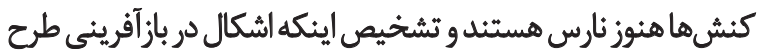

$$
\text { ناشى از كداميك است، مشكل بهنظر مئرسد. }
$$

در كروهى از كودكان معمولى مشاهده شده است كه درجه رسش

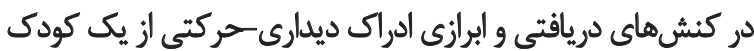

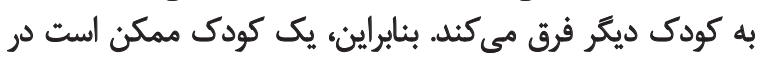

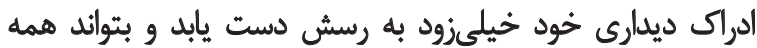

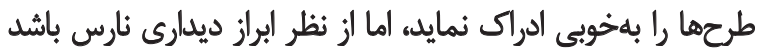

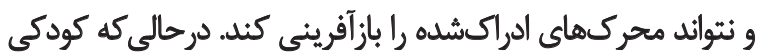

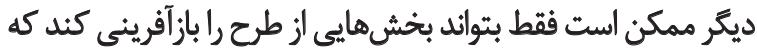

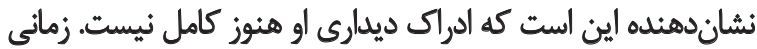

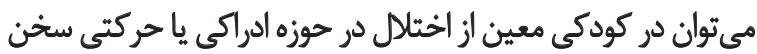

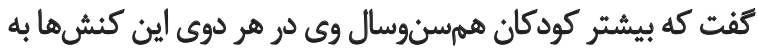

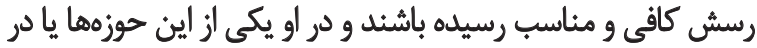

ارتباط مثقابل ميان آنها نارسايى ديده شود [1 1 ].] همان كونه كه در بخش نتايج (جدول شماره f) مشاهده شد،

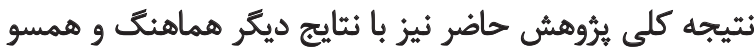

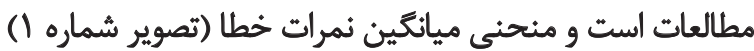

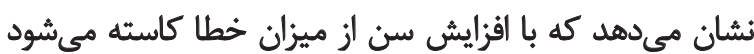

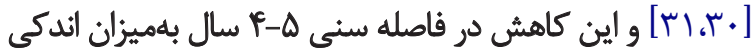

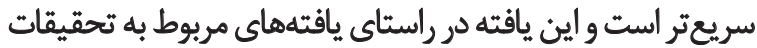

جنسيت مذكر در بروز خطاهاى ادامه كارى در طرح و و ه ه و تركيب

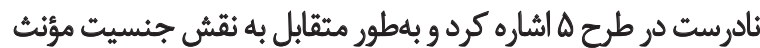

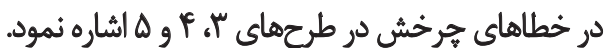

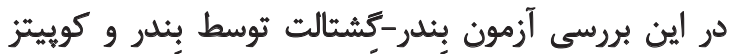

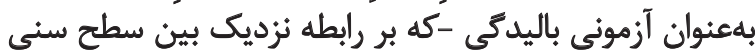

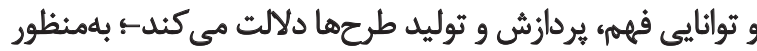

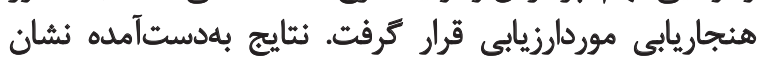

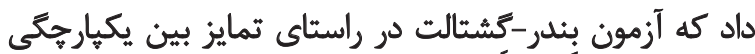

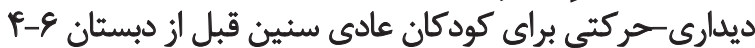

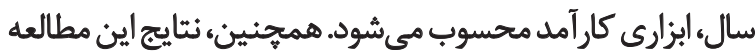
مؤيد و حامى اهداف و سؤالات يُروهش بودي

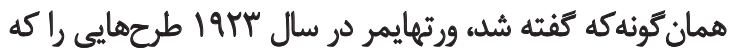

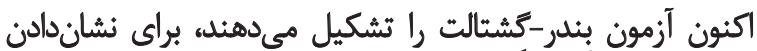

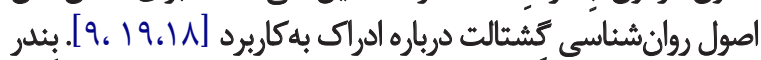

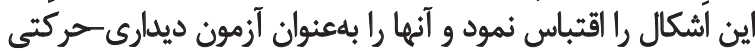

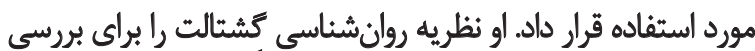
شخصيت وفعاليتهاي بالينى مورداستفاده قرار داد داد.

بندر عنوان كرد كه ادراى و بازآفرينى اشكال كُشتالت توسط

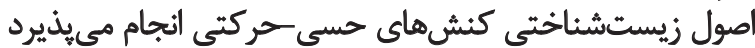

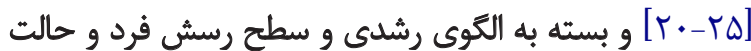

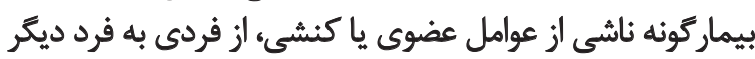

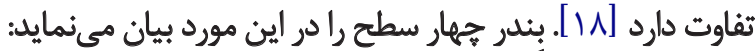

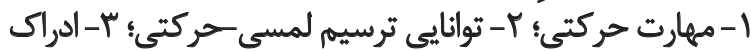

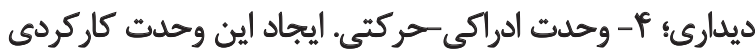

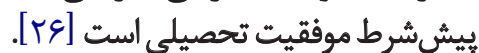
درنهايت بندر نتيجه كرفت كه بازآفرينى طرحماي كُشتالت

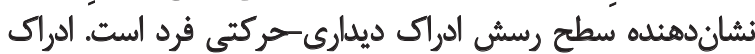

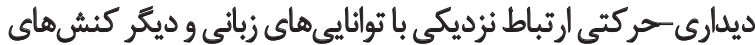

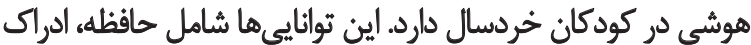




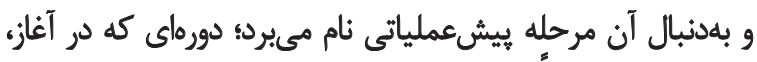

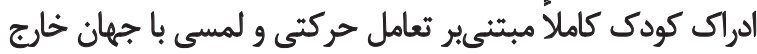

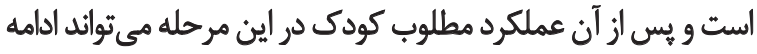

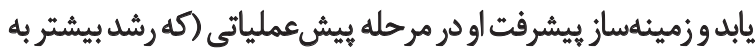
جنبهاي نمادين و زبانى مربوط است) شود.

با مشاهده تعداد خطاها و الكوهاى ترسيم ثبتشده در كروههاى

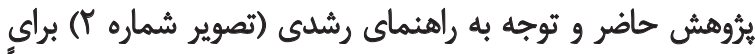

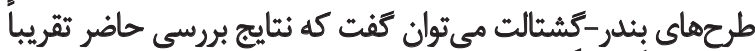

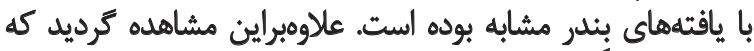

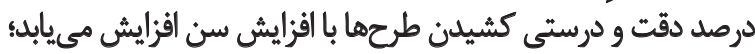

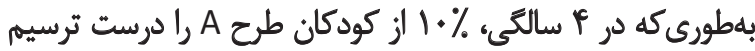

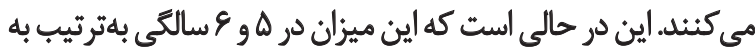

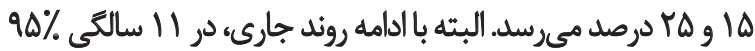

$$
\text { از كودكان از عهده ترسيم اين شكل برمى آيند. }
$$

با إين حال روند كاهشى خطاها ثابت و يكنواخت نيست و سرعت

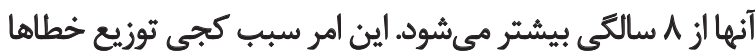

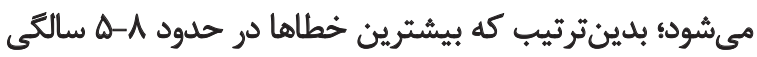

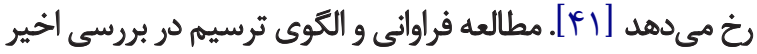

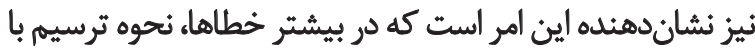

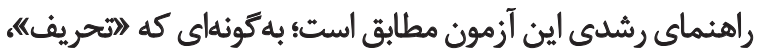

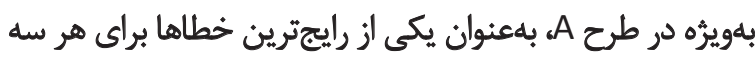

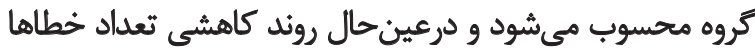

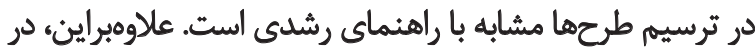

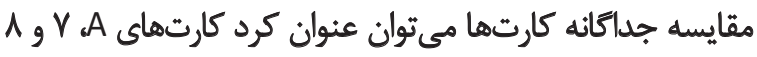
درمجمع دشوارترين كارتها بهشمار مى آيد.

تئيجنيرى

توجيهات موجود درباره خطاهاى مطرحشده مي تواند به دلايل

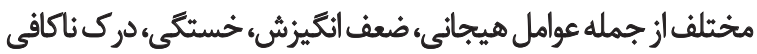

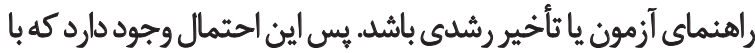

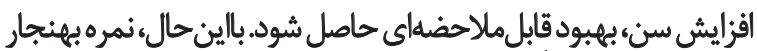

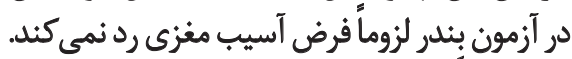

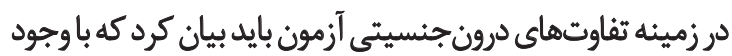

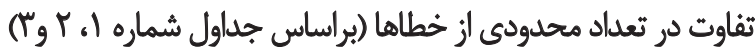

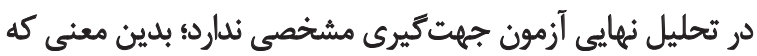

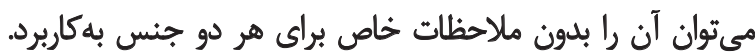

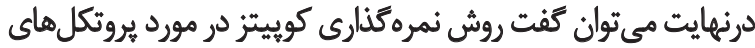

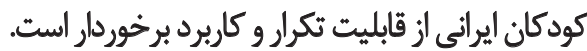

يشينهادات

ازآنجاكه شركتكئدكان در اين يثروهش به دامنه يك سال

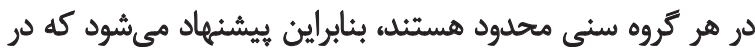

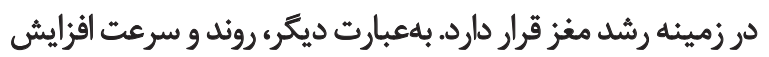

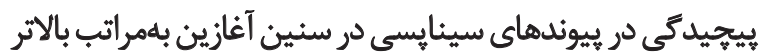

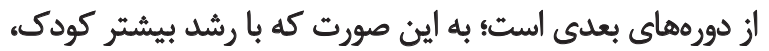

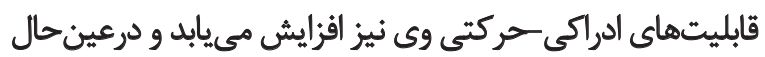

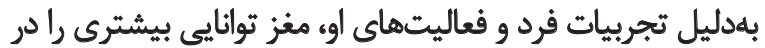

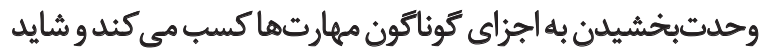
اين افزايش در سنين آغازين با سرعت بالاترى باشدائ

يافتهاى الشإير" و "اسيميسون" (1990) نشاندهنده اين بود كه

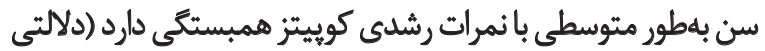

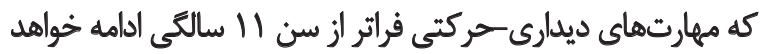

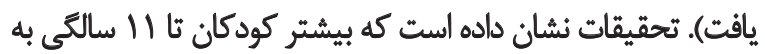

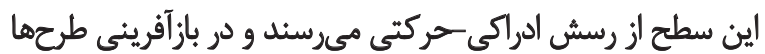
خطايى مرتكب نمى أشوند [اسب].

در ديكر مطالعات مشابه، البريتولو وممكارانش بهاين نتيجه رسيدند

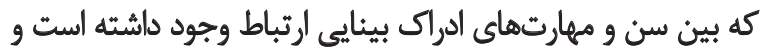

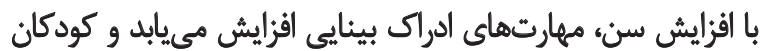

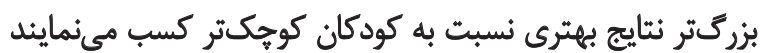

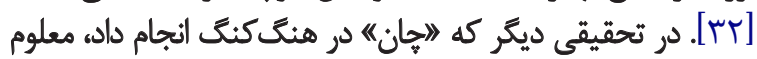

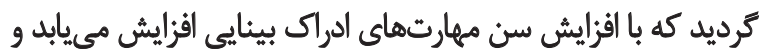

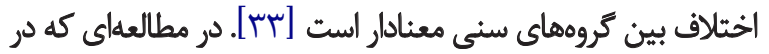

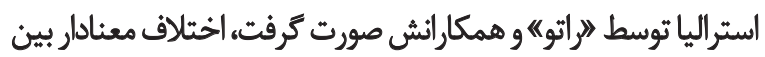

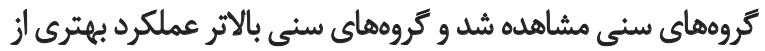

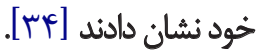

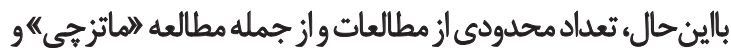

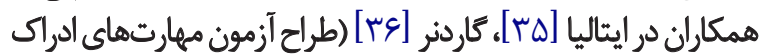

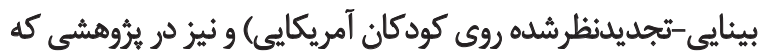

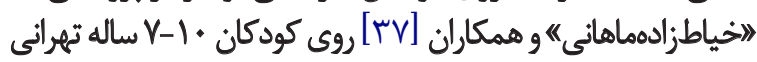

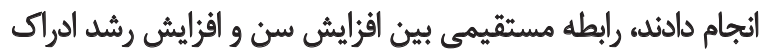

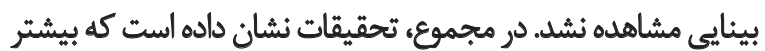

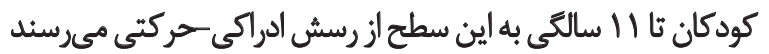
ودر بازآفرينى طرحها خطايى مرتكب نمى سورند.

"ابيلون" و همكارانش اشاره مى كنند كه رشد ديدارى حركتى تا

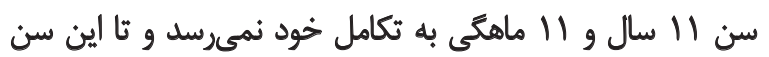

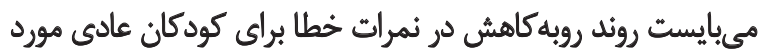

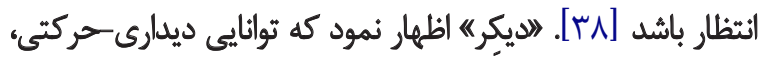

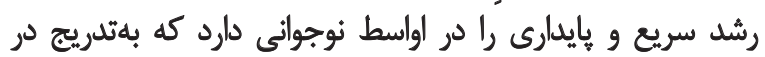

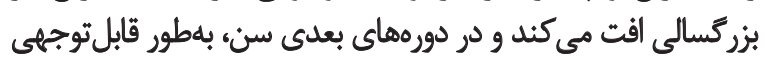

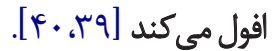

بِندر تبيين مينمايد كه الكوبردارى تصاوير آزمون بِندر، بازتابي از

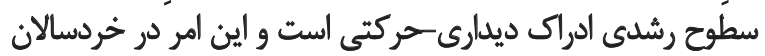

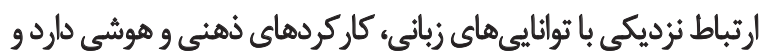

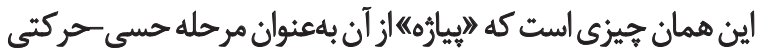




\section{References}

[1] Groth-Marnat G. Handbook of psychological assessment. [H. Pasha Sharifi, Persian trans]. $1^{\text {st }}$ ed. Tehran: Roshd Publication; 1995, p. 331-379.

[2] Alilou MM. [Standardization of the bender-gestalt test on 7 to 10 year-old school children in Tabriz (Persian)]. Psychological Research. 1994; 2(1):33-45.

[3] Tirgari A. [Brain disease, psychotic and normal in Sari city (Persian)]. Journal of Medical Science of Mazandaran Univercity. 1997; 6(12):36-43

[4] Kapaln H, Sadock B. Handbook of psychiatry. [NA. Pourafgari, Persian trans]. $2^{\text {nd }}$ ed. Tabriz: Azadeh Press; 1995, p. 367-365.

[5] Abdollahi I, Joghataee MT, Salavati M, Ebrahimi E, Ashayeri H. [Explicit learning of cognitive skills-motor, after stroke using the affected hand (Persian)]. Journal of Rehabilitation. 2008; 8(3):2430

[6] Groth-Marnat G. Handbook of Psychological Assessment. New York: van Nostrand Rinhild; 1984, p. 91-113.

[7] Lachapelle J, Ouimet C, Bach M, Ptito A, Mckerral M. Texture segregation in traumatic brain injury: A vep study. Vision Research. 2004; 44(24):2835-42.

[8] Silverstein SM, Keane BP. Perceptual organization impairment in schizophrenia and associated brain mechanisms: Review of research from 2005 to 2010. Schizophrenia Bulletin. 2011; 37(4):690-699.

[9] Koppitz EM. The bender-gestalt test for young children. New York: Grune \& Stratton; 1963, p. 15-35.

[10] Ghasemzadeh H. A Pilot study of the bender-gestalt test in the sample of Iranian normal children. Journal of Clinical Psychology. 1988; 44(5):787-92.

[11] Lacks P. Bender gestalt screening for brain dysfunction. $2^{\text {nd }} \mathrm{ed}$. Hoboken, N.J.: John Wiley \& Sons Inc; 1999.

[12] Marley ML. Organic brain pathology and the bender gestalt test: A differential diagnostic scoring system. New York: Grune \& Stratton; 1982, p. 19-60.

[13] Rosini ED, Kaspar JC. Validity of the bender gestalt emotional indicators. Journal of Personality Assessment. 1987; 51(2):254-61.

[14] Oas P. Validity of the draw-a-person and bender gestalt test as measures of impulsivity with adolescents. Journal of Consulting and Clinical Psychology. 1984; 52(6):1011-19.

[15] Hutt ML. The hutt adaptation of bender gestalt test. $4^{\text {th }}$ ed. New York: Grune \& Stration; 1985.

[16] Watkins CE, Campbell VL, Nieberding R, Hallmark R. Contemporary practices of psychological assessment by clinical psychologists. Professional Psychology: Research and Practice. 1955; 26(1):54-60.

[17] Nealm MD, Mckay MF. Scoring the bender gestalt test using the koppitz developmental system: interrater reliability, item difficulty, and scoring implications. Perceptual and Motor Skills. 1985; 60(1):627-636.

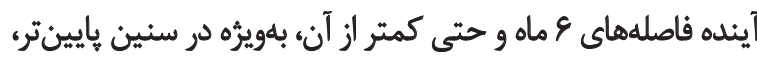

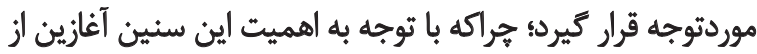

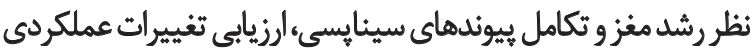

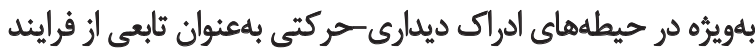

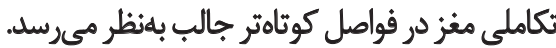

$$
\text { ن }
$$

دريايان محققان بر خود لازم مي دانند تامراتب تشكرو قدرداني خود

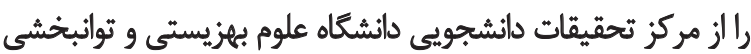

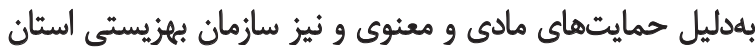

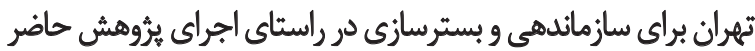

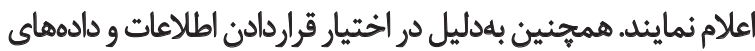

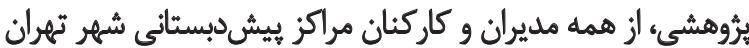

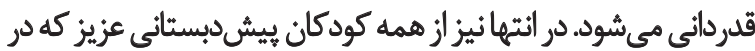

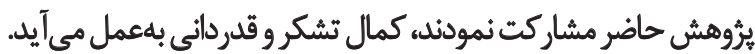


[18] Wagemans J, Feldman J, Gepshtein S, Kimchi R, Pomerantz JR, van der Helm PA, et al. A century of Gestalt psychology in visual perception: II. Conceptual and theoretical foundations. Psychological Bulletin. 2012; 138(6):1218-52.

[19] Wagemans J, Elder JH, Kubovy M, Palmer SE, Peterson MA, Singh M, et al. A century of gestalt psychology in visual perception: perceptual grouping and figure-ground organization. Psychological Bulletin. 2012; 138(6):1172-1217.

[20] Anzai A, Peng X, van Essen DC. Neurons in monkey visual area V2 encode combinations of orientations. Nature Neuroscienc. 2007; 10(10):1313-21.

[21] Hedgé J, van Essen DC. Selectivity for complex shapes in primate visual area V2. Journal of Neuroscience. 2000; 20(5):61-67.

[22] Hess RF, May KA, Dumoulin SO. Contour integration: Psychophysical, Neurophysiological and Computational Perspectives. In: Wagemans J, editor. Oxford Handbook of Perceptual Organization. Oxford: Oxford University Press; 2013, p. 189-206.

[23] Ito M, Komatsu H. Representation of angles embedded within contour stimuli in area V2 of Macaque monkeys. Journal of Neuroscience. 2004; 24(13):3313-24.

[24] Pasupathy A, Connor CE. Responses to contour features in Macaque area V4. Journal of Neurophysiology. 1999; 82(5):2490502.

[25] Ledgeway T, Hess RF, Geisler WS. Grouping local orientation and direction signals to extract spatial contours: Empirical tests of "association field" models of contour integration. Vision Research. 2005; 45(19):2511-22.

[26] Buros O, Krisen ED. The eighth mental measurement year book. New York: Gryphon Press; 1978.

[27] Vancleef K. Psychophysical and neuropsychological perspectives on perceptual organization with a focus on texture and contour processing $[\mathrm{PhD}$ thesis]. Leuven: Katholieke Universiteit Leuven Laboratory of Experimental Psychology; 2013.

[28] Bender L. A visual motor test and its clinical use. Research monograph. New York: American Orthopsychiatry Iatric Association; 1938.

[29] Alizadeh Zarei M, Bayrami M, Mahmoodalilou M, Hashemi T. [Perceptual organization and central coherence during the visual processing in children with autism: evidence for disrupted functional connectivity in autistic brain (Persian)]. Journal of Rehabilitation. 2014; 15(2):12-21.

[30] Kushesh MR, Bahrami H, Forughoddin Adl A, Same Siahkalhoroudi L, Karimi H. [Comparison of visual perception discrimination between the three groups of normal children, mentally retarded and learning disorders being orphaned covered in Tehran (Persian)]. Journal of Rehabilitation. 2007; 7(4):52-56.

[31] Shapiro SK, Simpson RG. Koppitz scoring system as measure of bender-gestalt performance in behaviorally and emotionally disrupted adolescents. Journal of Clinical Psychology. 1995; 51(1):108-112.

[32] Brito GN, Santos TR. The bender-gestalt test for 5 to 15 years old Brazilian children norms and validity. Brazilan Journal of Medical Biology Research. 1996; 29(11):1513-18.
[33] Chan PW. Comparison of visual motor development in Hong Kong and USA assessed on the qualitative scoring system for the modified bender-gestalt test. Psychological Reports. 2001; 88(1):236-40.

[34] Rateau F, Laumonier B, Hyndman RJ. Normative data for the rosner test of visual analysis skills on an Australian population. Optometry and Vision Scince. 2003; 80(6):431-436.

[35] Mazzeschi C, Lis A. The bender gestalt test Koppitzs developmental scoring system administrated to two samples of Italian preschool and primary school children. Perceptual and Motor Skills. 1999; 88(3):1235-44.

[36] Gardner MF. Test of visual-perceptual skills (non-motor)-revised manual. $1^{\text {st }}$ ed. California: Psychological and Educational Publication Inc; 1996.

[37] Khayatzadeh Mahani M, Mardani Shahrbabak B, Gholamian H, Rahgozar M, Soroory M, Fadaie F. [Visual perceptual skills in normal children aged 7 to 13 years in Tehran city (Persian)]. Journal of Rehabilitation. 2011; 11(4):8-14.

[38] Bolen LM, Hewett JB, Hall CW, Mitchell CC. Expended kopitz scoring system of bender gestalt visual-motor test for adolescents: A pilot study. Psychology in the Schools. 1992; 29(1):113-15.

[39] Decker SL. Measuring growth and decline in visual-motor processes with the bender gestalt second edition. Journal of Psychoeducational Assessment. 2008; 26(1):3-15.

[40] Rajabi G. Normalizing the bender visual-motor gestalt test among 4-7 year-old children. Journal of Applied Science. 2009; 9(6):1165-1169.

[41] Taylor RL, Kauffman D, Partenio I. The koppitz developmental scoring system for bender gestalt: Is it developmental? Psychology in the Schools. 1984; 21(1):425-28. 